\title{
Female platelets are hard to control
}

\author{
Y. Appelman • R. J. de Winter
}

Published online: 18 October 2011

(C) The Author(s) 2011. This article is published with open access at Springerlink.com

In the Western world, cardiovascular disease (CVD) is the leading cause of death in women. In Europe, $43 \%$ of men die from CVD, compared with $54 \%$ in females, although there has been a decline in the absolute number of deaths from CVD in the past 30 years (in the Netherlands a $60 \%$ decline in men and $52 \%$ in women with correction for age distribution). Due to ageing of the population and the longer life expectancy of women, it is to be expected that even more women will die from CVD than men in the future.

At younger age (pre-menopausal), females are more susceptible to smoking-related damage when compared with man, possibly due to the downregulation of oestrogendependent vasodilatation of the endothelial wall of the artery. As a consequence, smoking is the leading cause of ischaemic heart disease at younger age in women. It is known that menopause leads to a transition in hormonal status and is associated with worsening of the cardiovascular risk profile. After menopause the characteristic risk factors for cardiovascular disease are similar in males and females; however, the impact of these factors does differ between the sexes. Especially hypertension, hypercholesterolaemia and diabetes have a higher prevalence and impact in postmenopausal women when compared with men.

However, besides the traditional overall risk factors, women have female-specific risk factors which have a high

\footnotetext{
Y. Appelman $(\bowtie)$

Department of Cardiology, VU University Medical Center,

De Boelelaan 1117,

1081 HV Amsterdam, the Netherlands

e-mail: y.appelman@vumc.nl

R. J. de Winter

Department of Cardiology, Academic Medical Center,

Meibergdreef 9 ,

1105 AZ Amsterdam, the Netherlands
}

influence on the occurrence of CVD and their outcome after coronary interventions. Women with a history of diabetes or hypertensive disease in pregnancy (especially pre-eclampsia) or with premature menopause are at increased risk for CVD later in life. But, these factors are not taken into account in clinical trials which leads to underestimation of the risk profile of females [1].

Furthermore, with respect to the pathophysiology, women differ from men. Women have less obstructive coronary artery disease along the entire spectrum of acute coronary syndromes (ACS) and across all age groups. Even in early stages females have a lower atheroma burden and different plaque morphology than men. This phenomenon is known as the 'gender paradox' as women do have symptoms and die from myocardial infarction, but have apparently less extensive disease. It is hypothesised that different mechanisms play a role with respect to the pathophysiology of atherosclerosis and the occurrence of ACS in both sexes. In women, abnormal endothelial function, difference in pre- and post-menopausal vascular repair (oestrogen levels are changing), a higher incidence of microvascular disease and a difference in autonomic function (women have higher parasympathetic cardiac activity while men have a higher sympathetic cardiac activity) are mentioned as a possible explanation in this matter [2].

In this issue of the journal, Breet et al. address the potentially important issue of differences in platelet physiology between the genders [3]. Previous studies have suggested that women have a higher magnitude of platelet reactivity when compared with men. In addition, on-treatment platelet reactivity with aspirin is higher in women. It is known that platelet reactivity plays a key role in atherosclerosis and thrombus formation. Therefore, (dual) antiplatelet therapy is the treatment of choice for 
patients undergoing elective coronary stent implantation and in those presenting with ACS. It is suggested that the higher platelet reactivity in women might be one of the explanations for the higher incidence of death in female patients with CVD. Furthermore, studies have suggested that women do not have comparable benefit from antithrombotic therapy [4]. In their study, Breet at al. compared the magnitude of on-treatment platelet reactivity between genders in patients on dual antiplatelet therapy undergoing elective coronary stenting [3]. This study was a subanalysis of the POPular study (The Do Platelet Function Assays Predict Clinical Outcomes in Clopidogrel Pretreated Patients Undergoing Elective PCI study) which was a prospective, observational study including consecutive patients with established coronary artery disease scheduled for elective coronary stent implantation. It was concluded that patients exhibiting a high on-treatment platelet reactivity status may be at higher risk for adverse events post-PCI. In the subanalysis, two platelet function tests were used: the Light Transmission Aggregometry (LTA) and the VerifyNow, two of the well-established tests for platelet reactivity. As mentioned in prior studies, they found that women (234 patients) had a higher magnitude of on-aspirin and on-clopidogrel platelet reactivity at baseline compared with men (717 patients), even after adjustment for potential confounders in multivariate analysis. However, the occurrence of the composite endpoint (death, myocardial infarction, stent thrombosis and stroke) was similar between the two groups. Consequently, they concluded that with respect to this result it seemed unlikely that the difference in platelet reactivity accounts for a worse prognosis in women. Although this study was well designed and performed by a highly experienced research group, a few comments can be made [5].

In this study, women have a higher magnitude of onaspirin and on-clopidogrel platelet reactivity but the absolute difference in clinical endpoints is rather small and therefore does not seem to be clinically relevant $(22.4 \pm$ $11.4 \%$ female vs. $19.8 \pm 11.1 \%$ male on-aspirin LTA and $59.6 \pm 13.2 \%$ female vs. $56.9 \pm 14.6 \%$ male on-clopidogrel LTA). These findings are not new and large randomised secondary prevention trials have demonstrated a worse outcome in women on dual antiplatelet therapy (CURE, CAPRIE, CREDO trials). Interestingly, this outcome seems to change somewhat in favour of women when treated with the newer antiplatelet drugs as prasugrel and ticagrelor (PLATO, TRITON trials). This suggests that the biological availability of the drug plays an important role. As hormonal changes influence the gastrointestinal uptake of antiplatelets and also their metabolism, these new drugs seem to be of clinical importance.
Furthermore, the study is based on a subgroup analysis using a different endpoint and consequently is not powered for comparison of the magnitude of on-treatment platelet reactivity between genders.

Finally, the study does not indicate whether measuring on-treatment platelet reactivity in patients receiving antiplatelet therapy is helpful and whether women might particularly benefit from such measurements. According to the recent ESC nSTE-ACS guidelines, personalised antiplatelet treatment has yet not arrived in the clinical arena, as platelet function testing received a $\mathrm{IIb}$ recommendation [6].

In conclusion, the data reported in this study are not sufficient to answer the questions with respect to the relation between gender, the occurrence of clinical endpoints and platelet reactivity in patients undergoing elective PCI and using dual antiplatelet therapy. The higher platelet activity in females and the possible consequences is hard to understand and further large randomised clinical trials with the new-generation antiplatelet therapy are needed to establish their role in coronary artery disease. However, this study again points out clearly that women are hard to control.

Open Access This article is distributed under the terms of the Creative Commons Attribution Noncommercial License which permits any noncommercial use, distribution, and reproduction in any medium, provided the original author(s) and source are credited.

\section{References}

1. Maas AH, Appelman YE. Gender differences in coronary heart disease. Neth Heart J. 2010;18:598-602.

2. Vaccarino V, Badimon L, Corti R, et al. Ischemic heart disease in women: are there sex differences in pathophysiology and risk factors. Position Paper from the Working Group on Coronary Pathophysiology and Microcirculation of the European Society of Cardiology. Cardiovasc Res. 2011;90:917.

3. Breet NJ, Sluman MA, van Berkel MAJPJ, et al. Effect of gender difference on platelet reactivity. Neth Heart J 2011;19:xx-xx.

4. Berger JS, Bahtt DL, Cannon CP, et al. The relative efficacy and safety of clopidogrel in women and men. A sex-specific collaborative meta-analysis. J Am Coll Cardiol. 2009;54:1935-45.

5. Becker DM, Segal J, Vaidya D, et al. Sex Differences in Platelet Reactivity and Response to Low-Dose Aspirin Therapy. JAMA. 2006;295:1420-7.

6. Hamm CW, Bassand J-P, Agewall S, et al. ESC Guidelines for the management of acute coronary syndromes in patients presenting without persistent ST-segment elevation. The Task Force for the management of acute coronary syndromes (ACS) in patients presenting without persistent ST-segment elevation of the European Society of Cardiology (ESC) Eur Heart J doi:10.1093/eurheartj/ ehr236 